\title{
EL ANALFABETISMO EN HONDURAS
}

\section{Por: Sandra Flores. Auxiliar de Investigación}

Instituto de Investigación Jurídica, Universidad Nacional Autónoma de Honduras.E-Mail:sandra.flores@unah.edu.hn

\section{Resumen}

Es prioridad en estos momentos dar a conocer la situación tan agravante como deficiente que enfrenta el sistema educativo nacional como uno de los entes más importantes que enlistan las causas que contribuyen a los altos grados de analfabetismo que Honduras está confrontando en todas las áreas. Es por ello que hemos retomado el tema porque nos parece preocupante que cada día la Educación en Honduras no va de acuerdo con la curricula básica nacional, el grado de personas analfabetas ha incrementado. Al realizar el presente estudio y verificar las estadísticas proporcionadas por el Instituto Nacional de Estadísticas (INE) cerca de 804,600 mil personas de los 8.093,877 millones de habitantes que tiene Honduras son analfabetas es alarmante que más del 14.9 por ciento de la población no tiene acceso a la educación; otra causa por cual se genera la falta de conocimiento de dicha materia es el elevado índice de desempleo y descomposición familiar. Con el presente trabajo se pretende que sirva de referencia para buscar propuestas y soluciones ya que hemos Ilegado hasta el fondo de la problemática, situación, por lo cual es necesario que se reflexione y se tome conciencia que las consecuencias son agudizantes y que somos parte de ella.

Palabras clave: - Analfabetismo, Educación, Honduras

\section{Abstract}

The priority now is to present the aggravating and deficient situation the national education system is facing as one of the most important entities. Listing the causes that contribute to high levels of illiteracy that Honduras is facing in all areas. That is why we have taken up the issue because it seems that every day Education in Honduras does not agree with the national basic curriculum. The degree of illiterate people has increased. In conducting this study and verify the statistics provided by the $\mathrm{Na}$ tional Research Institute (INE) about 804.600 people in $8,093,877$ people who are illiterate in Honduras is alarming that more than $14.9 \%$ percent of the population does not have access to education. Another cause which is generated by the lack of knowledge of the matter is the high unemployment and family breakdown. The present work is intended as a reference to search for proposals and solutions since we have reached the bottom of the problematic situation, which is necessary to reflect and become aware that the consequences are heightened and we are part of it

Keywords: Illiteracy, Educatión, Honduras 


\section{INTRODUCCIÓN}

A partir de la problemática del analfabetismo en nuestro país, es importante investigar acerca de cómo este afecta en gran medida el desarrollo cultural, social y tecnológico de nuestra sociedad Hondureña. Conforme a las estadísticas porcentuales proporcionadas, el analfabeto se encuentra en los estratos más pobres y vulnerables de nuestra sociedad, debido a que no ha podido acceder a un digno sistema educativo. Consideramos también que influye en gran parte el alto porcentaje de desempleo y desocupación.

En Honduras, la tasa de analfabetismo es elevada y llega al 15.2\% de la población mayor de 15 años de edad, resultando como de los peores problemas del cual se acarrea miseria, pobreza y la desesperanza; de acuerdo a datos estadísticos que maneja el Instituto de Nacional de Estadísticas (INE), no ha sido posible implementar un proceso de reforma educativa que en verdad produzca cambios que impacten en el desarrollo nacional; los proyectos más significativos han sido aquellos orientados a la calidad del proceso pero han estado en un eterno descuido y desinterés por causas de un paro o huelga en la educación.

En la coyuntura actual, preocupa mucho la confrontación entre las autoridades educativas y los docentes hondureños este es un detonante peligroso que atenta contra la educación por lo cual la población se encuentra subsumida en la ignorancia.

Según algunos informes, la educación juega un papel clave para el ejercicio de la democracia, el progreso y el bienestar individual colectivo, para que las nuevas generaciones puedan enfrentar con éxito los retos del nuevo milenio. Levantemos el perfil en la formación de las presentes y futuras generaciones porque en la educación esta nuestro futuro. 


\section{CAPÍTULO I}

\section{A.- ANALFABETISMO EN EL MUNDO}

El grave problema del analfabetismo en los países desarrollados y subdesarrollados sigue siendo uno de los temas prioritarios a solucionar desde que se analizara profundamente en la Conferencia Mundial de Educación para Todos, celebrada en 1990 en la ciudad de Jomtiem (Tailandia). En esta cita a la que asistieron representantes de países de todo el mundo se llegó al consenso de que la alfabetización es uno de los factores clave para resolver muchos de los problemas más acuciantes en todas las sociedades, que la realización plena del ser humano sólo se logra a través de la educación y que promover la educación es fundamental para el desarrollo de las naciones. No en vano constituye una herramienta muy eficaz para combatir la pobreza y la desigualdad, mejorar los niveles de salud y bienestar social, y sentar las bases para un crecimiento económico sostenido y una democracia duradera. Razón por la que la educación se incluyó entre los ocho Objetivos de Desarrollo del Milenio de las Naciones Unidas, fijándose el año 2015 como fecha límite para alcanzar un 100\% de educación primaria para todos los niños del mundo. Como consecuencia, a finales del año 2001 la Asamblea General de la ONU proclamó al período 2003-2012 como el 'Decenio de las Naciones Unidas de la Alfabetización', ratificando así el objetivo fijado en el Foro de Educación Mundial de Dakar de educación primaria efectiva para el año 2015.

Pero la situación más grave se deriva del retroceso que ha experimentado el proceso de alfabetización en el mundo; mientras que en la mitad del siglo XX se habían logrado grandes avances en términos de la erradicación de analfabetismo, objetivo largamente declarado por la comunidad internacional actualmente parece que esta tendencia está cambiando y se teme que hasta podrían perderse los logros obtenidos en el pasado ante el recorte de recursos para la educación en muchos países castigados por crisis económicas. En estos momentos, más de un 15\% de la población mundial es analfabeta y por lo tanto excluida de cualquier posibilidad de participar activamente en la sociedad del conocimiento. Son pueblos enteros los que sufren las consecuencias de la imposibilidad de acceder a la herramienta más básica para comunicarse en la sociedad moderna. Se vulnera así el derecho de la educación básica, un derecho formalmente reconocido desde su consagración hace 50 años en la Declaración Universal de los Derechos Humanos. (Comisión Nacional para el Desarrollo de la Educación Alternativa no Formal (CONEANFO 2011). 


\section{B.- ANALFABETISMO EN HONDURAS}

El sistema educacional de Honduras es el más atrasado de Centroamérica pues apenas 32 de cada 100 estudiantes logran terminar la primaria sin repetir grados. Datos del Programa para el Desarrollo de la Organización de Naciones Unidas (PNUD) revelan que el $51 \%$ de los matriculados termina la primaria con un promedio de 9,4 años y que los niveles de deserción escolar cada vez son más elevados.

El más agudo problema es que el sistema educacional básico sólo cubre al 86,5\% de quienes están en edad escolar, mientras el 13,5\% restante no puede acceder a la enseñanza. Aunque la Constitución hondureña estipula de modo formal que los menores deben ser atendidos por los servicios educativos, muchos arriban a la mayoría de edad analfabetos, mientras el Estado trata de justificarse con la insuficiencia de los recursos a su alcance. El analfabetismo abarca a más de medio millón de personas en este país, es decir, casi el equivalente de toda la población mayor de 15 y menor de 40 años. Las fuentes agregan que el problema se agudiza ante la escasez de recursos públicos y una insuficiente y poco equitativa oferta en el orden educacional, tanto en cantidad como en calidad. También la necesidad de mejorar los ingresos familiares obliga a muchos niños a dejar la escuela para dedicarse al trabajo, generalmente de forma permanente. A esto se une el bajo nivel educativo alcanzado por muchos padres, por similares razones, y las condiciones precarias en que vive más del $80 \%$ de los hondureños.

En 10 de los 18 departamentos en que se encuentra dividido este país, más del 9\% de los estudiantes se ven precisados a repetir grados. Las cifras acumuladas por el ministerio del ramo reflejan que ningún departamento de Honduras alcanza, como promedio, los seis años mínimos de educación primaria. Según datos recientes de indicadores de eficiencia educativa, por cada mil alumnos matriculados en el primer grado en 1990, sólo 292 (29\%) completaron la escuela primaria en seis años y 468 , es decir, un 46\%, no lograron Ilegar al final. Mucho más preocupante resulta el panorama universitario, en el cual la tasa de aprobados no logra sobrepasar el $20 \%$, incluso, en centros como la Universidad Nacional Autónoma de Honduras (UNAH). Evaluaciones realizadas por organismos internacionales denuncian el atraso de la inversión estatal en el sector con respecto a la mayoría de los países de la región y que el modelo educativo vigente desde hace más de una década en Honduras ha llegado a sus límites. (Comisión Nacional para el Desarrollo de la Educación Alternativa no Formal (CONEANFO 2011). 


\section{CAPITULO II}

\section{A.- CONCEPTUALIZACIÓN}

El Analfabetismo es la incapacidad que posee el ser humano de leer y escribir, que se debe generalmente a la falta de aprendizaje.

El analfabetismo aparece como resultado de la falta de educación y si bien el porcentaje de la población mundial aún sumida en tales condiciones es infinitamente menor a otras épocas de la historia, todavía hay numerosas sociedades y comunidades que cuentan con gran parte de analfabetos en su población; es considerado uno de los principales problemas y deudas de la humanidad ya que aquellas personas consideradas analfabetas no lo son por opción propia si no por la existencia de altos niveles de pobreza, miseria y falta de oportunidades educativas en el medio en el que se encuentran insertas. Tal es así que las tasas de analfabetismo se hacen claramente visibles en países en vías de desarrollo en los cuales los sistemas educativos son deficientes o directamente no son prioridad.

\section{B.- TIPOS DE ANALFABETISMOS MÁS CONOCIDOS}

La crisis de la educación se manifiesta en los tres niveles de analfabetismo: el analfabetismo absoluto, el analfabetismo funcional y el analfabetismo moral. De manera típica decir que una persona es analfabeta significa que la persona no puede leer ni escribir. Pero la palabra tiene otros sentidos. Algunas veces se usa con respecto a alguien que es ignorante de los fundamentos de un arte particular o de un área del conocimiento. Es este significado más amplio el que está en vista cuando, por ejemplo, decimos que una persona es analfabeta con respecto a la música. La palabra también puede usarse para describir a una persona que se queda corta con respecto a algún estándar esperado de competencia sobre alguna habilidad o cuerpo de información.

Describiremos los tipos de analfabetismo:

B.1.- ANALFABETISMO ABSOLUTO. Es la incapacidad de leer y escribir, que se debe generalmente a la falta de aprendizaje.

B.2.- ANALFABETISMO FUNCIONAL: Se denomina a la incapacidad de un individuo para utilizar su capacidad de lectura, escritura y cálculo de forma efi- 
ciente en las situaciones habituales de la vida. Se diferencia del analfabetismo en sentido estricto en que éste supone la incapacidad absoluta de leer o escribir frases sencillas en cualquier idioma.

B.3.- ANALBAFETISMO MORAL: Aunque es difícil para algunas personas creer que alguien involucrado en la educación vaya a actuar intencionalmente en maneras que induzcan al analfabetismo funcional. Muchos educadores han dado su cuota de contribución al analfabetismo moral de sus estudiantes. Los prejuicios contra los valores religiosos y morales nos han dejado con una generación de analfabetos morales En generaciones pasadas los padres eran más diligentes en traspasar sus principios y valores a sus hijos y eran ayudados por las iglesias y las escuelas que enfatizaban la educación religiosa y moral. En años recientes, en contraste, nuestra sociedad se ha convertido en una sociedad más y más secular y el currículo de las escuelas públicas ha sido despojado de casi todo contenido ético. Como resultado las universidades deben confrontar a un cuerpo de estudiantes ignorantes de la evidencia y de los argumentos que subyacen y apoyan muchos de nuestros principios morales y prácticas tradicionales. Esta pérdida del orden moral está vinculada inseparablemente a la ruina de nuestra tradición intelectual.

B.4.- ANALFABETISMO TECNOLOGICO: Este es un nuevo tipo y no está relacionado con la falta de instrucción en las disciplinas más básicas (como la lectura, escritura y las reglas matemáticas elementales). El analfabetismo tecnológico se refiere a la incapacidad para utilizar la nueva tecnología tanto en la vida diaria como en el mundo laboral y no está reñido con la educación académica en otras materias, es decir que una persona puede ser bien preparada en distintas disciplinas educativas pero analfabeta en esta disciplina.

Las consecuencias de este nuevo fenómeno son muy variadas y, en realidad, la mayoría de ellas aún está por llegar. Desde dificultades para encontrar un empleo, hasta problemas para desenvolverse en la vida diaria. A medida que las nuevas tecnologías de la información se incorporen a nuestra forma de vida, el hueco existente entre los que saben aprovecharlas y los que no se irá ensanchando al mismo ritmo. De momento, el analfabetismo tecnológico se manifiesta únicamente en circunstancias concretas y relativamente aisladas: recién licenciados que no consiguen su primer empleo por no saber usar una computadora, directivos que ven peligrar su carrera por no saber aplicar las nuevas tecnologías a su negocio y cualquiera cuya calidad de vida pudiera mejorar en 
caso de sacarle mejor partido a las nuevas tendencias. Sin embargo, en un futuro no muy lejano, el analfabetismo tecnológico puede convertirse en un factor de mayor alcance y que, por ejemplo, ocasione importantes diferencias entre países o regiones "analfabeto tecnológico", independientemente de su nivel de educación e incluso de su clase social o su poder adquisitivo.

\section{CAPÍTULO III}

\section{CAUSAS QUE CONTRIBUYEN AL ANALFABETISMO EN HONDURAS}

A.- ASPECTO SOCIO-ECONOMICO. En lo que respecta al aspecto socio-económico, se presenta la persistencia de pobreza, generando esto, el atraso del desarrollo tecnológico y científico en nuestro país. La pobreza y el analfabetismo están muy ligados. Si bien la pobreza es una de las causas del analfabetismo, a la vez, es una consecuencia, pues provoca mayor pobreza manteniéndolos en esa condición. El conocimiento tiene un papel decisivo en el proceso de desarrollo; entonces, si no existe una buena educación, se estaría impidiendo que los niños, jóvenes y adultos de Honduras puedan desarrollar sus capacidades intelectuales y humanas adecuadamente.

B.- EL DESEMPLEO. Es uno de los grandes problemas característicos de nuestro país. Aquí en Honduras, este fenómeno se vio agravado por el huracán Mitch que casi destruyó la agricultura y la industria manufacturera, y se agudizo aún más después del golpe de estado del 28 de Junio del 2009, entre las causas que originan el desempleo, tenemos la poca formación profesional y la falta de una política de empleo en el país, tanto de parte del sector público como también del privado.

C.- DESIGUALDAD. Un segundo drama para la juventud en Honduras es vivir en una sociedad de extremas desigualdades y en la cual la crisis económica y social golpea de forma directa a la niñez y juventud. El impacto del modelo de ajuste neoliberal en las familias hondureñas sigue generando desintegración y abandono. El 2002 empujó a muchos pobres hacia la indigencia y a muchas familias de la clase media hacía la pobreza, en contraste con el enriquecimiento de un reducido sector de la sociedad, el desempleo, el subempleo, la inseguridad laboral, los bajos salarios, la devaluación diaria del lempira frente al dólar y el alza de precios de la canasta básica afectan gravemente a la familia hondu- 
reña, cuyos miembros casi nunca califican para trabajos que garanticen condiciones de vida con dignidad. La baja escolaridad del hondureño y hondureña promedio y la desintegración familiar con el consecuente número de madres solteras ha supuesto la incorporación creciente de mujeres al mercado informal y la oferta de trabajo mal remunerados, con jornadas intensivas, y escasas o ninguna garantía laboral.

D.- REPITENCIA EN EL NIVEL BÁSICO. La tasa de repitencia nacional para el nivel básico (del 1ero al 6to grado) en el país es de 5.0\% (año 2010). Entre tanto, la tasa de culminación en primaria es cerca del $90 \%$ y al final del sexto grado solo 2 de 3 estudiantes culminan el ciclo.

La limitación de los recursos asignados hacia la educación explica en parte esos débiles resultados, ya que da lugar a hacinamiento y a poca provisión de equipamiento escolar, aparte de no contar con adecuada capacitación para los maestros. En ese sentido, la educación pública se concentra en el sector primario, mientras que su participación es mucho menos relevante en el sector secundario y solo vuelve a crecer en el sector terciario. Por otro lado, cerca del $80 \%$ de los niños estudian en escuelas públicas en el nivel primario, mientras que en el nivel secundario ese porcentaje disminuye significativamente y solo Ilega al $20 \%$. La asignación hacia la educación pública ha sido cercana al 5.4\% del PIB durante los últimos años. (www.bcie.org./uploaded/content/ article/1944368211pdf).

E.- DESERCIÓN ESCOLAR. Se puede definir como "el hecho de que un alumno/a o un grupo de algunos abandonan parcialmente o totalmente la educación escolar". Este es un proceso complejo, que no sólo está referido a la escuela y que tampoco tiene que ver, de manera uní causal, con la pobreza. Los estudios disponibles sobre la deserción señalan que ocurre en relación con diversos ámbitos: se deserta, progresivamente, desde la escuela, desde la familia, desde el barrio, desde el grupo de iguales y finalmente, desde las leyes vigentes en un país. Por lo general, el primer hito desde el cual se deserta es la escuela; a partir de allí, se posibilitan otras deserciones. De allí la importancia de abordar la deserción escolar y las condiciones que la hacen posible. Para analizar esta problemática es necesario de mencionar sus causas como la falta de acceso a los medios de transporte por bajos ingresos económicos familiares, embarazos a temprana edad, la falta de interés, problemas familiares, desmotivación y fracasos escolares, las desigualdades del sistema oficial educativo en distintos 
sectores de la República de Honduras. Los ejes centrales del sistema educativo establecen el desarrollo de una educación pública como el espacio fundamental de construcción de la cultura, de transmisión de los valores, de aprendizaje de las normas, de aprendizaje de los lenguajes. Lo importante es analizar las causas del fracaso en cuestión y buscar las mejores soluciones posibles. En ocasiones el fracaso escolar no se debe al alumno, sino a la escuela, a la poca calidad de la educación, la formación del profesorado, el número de alumnos por aula, la dedicación. También es importante tener en cuenta que la capacidad y rendimiento del alumno no vienen determinados por su nivel intelectual sino también de otras circunstancias como el medio cultural, la salud, la personalidad. No todo el que repite, abandona o no obtiene la titulación prevista es ya un fracasado, por el contrario puede ser la forma de evitarlo, afianzando sus conocimientos o tomando un nuevo camino más acorde con sus posibilidades reales. Es pues que no existe un límite que marque con claridad los fracasos y los éxitos. Y con ello los niveles de analfabetismo que tanto afecta a nuestra sociedad hondureña se disminuirían cada vez más, permitiendo vencer ese obstáculo que no nos permite desarrollarnos como país. (Comisón Nacional para el Desarrollo de las Naciones Unidas. (PNUD), 2011)

\section{CAPÍTULO IV}

\section{ESTADISTICAS DE ANALFABETISMO EN HONDURAS}

El analfabetismo, es un problema muy importante en la realidad educativa del país. En Honduras para mayo del 2011, el 14.9\% de las personas mayores de 15 años, no saben leer ni escribir. Los datos indican que la tasa de analfabetismo continúa siendo mayor en la población del área rural con un $22.0 \%$ frente a un $7.3 \%$ en la urbana.

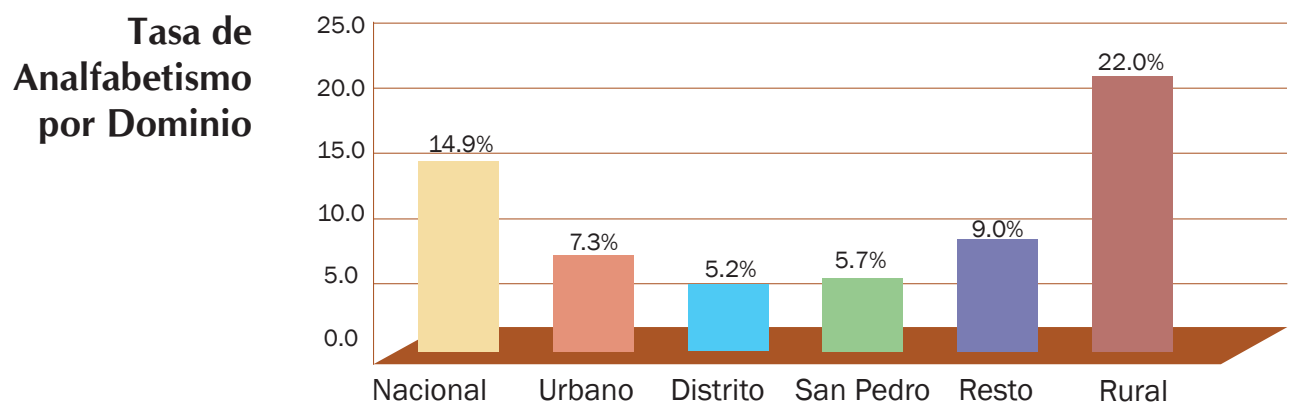




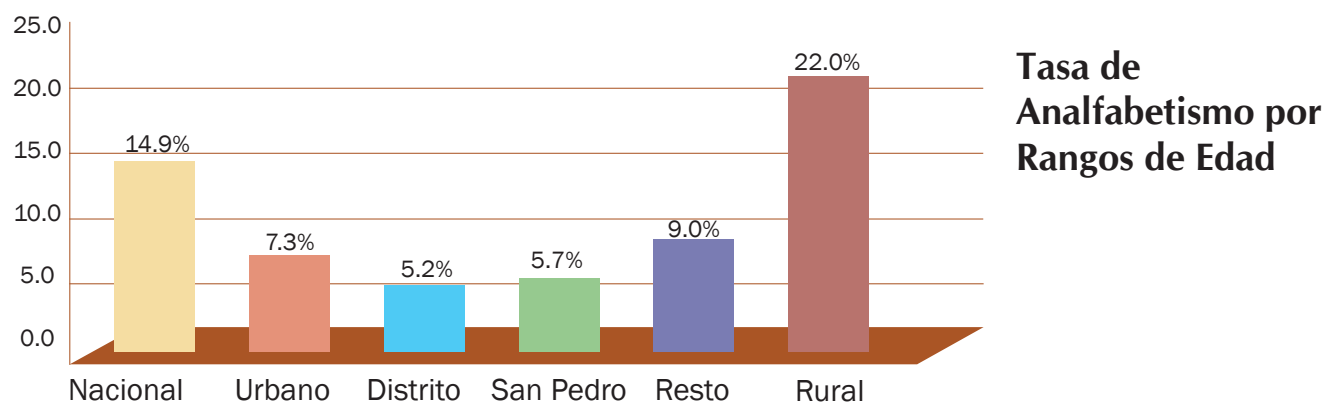

Además, el analfabetismo es superior en las personas de mayor edad. Hasta los 29 años la tasa es menor al 10\%, a partir de los 19 años aumenta hasta alcanzar su máximo en la población de 60 años y más, de la cual casi la mitad (47.9\%) no sabe leer ni escribir.

\section{Tasa de Analfabetismo total}

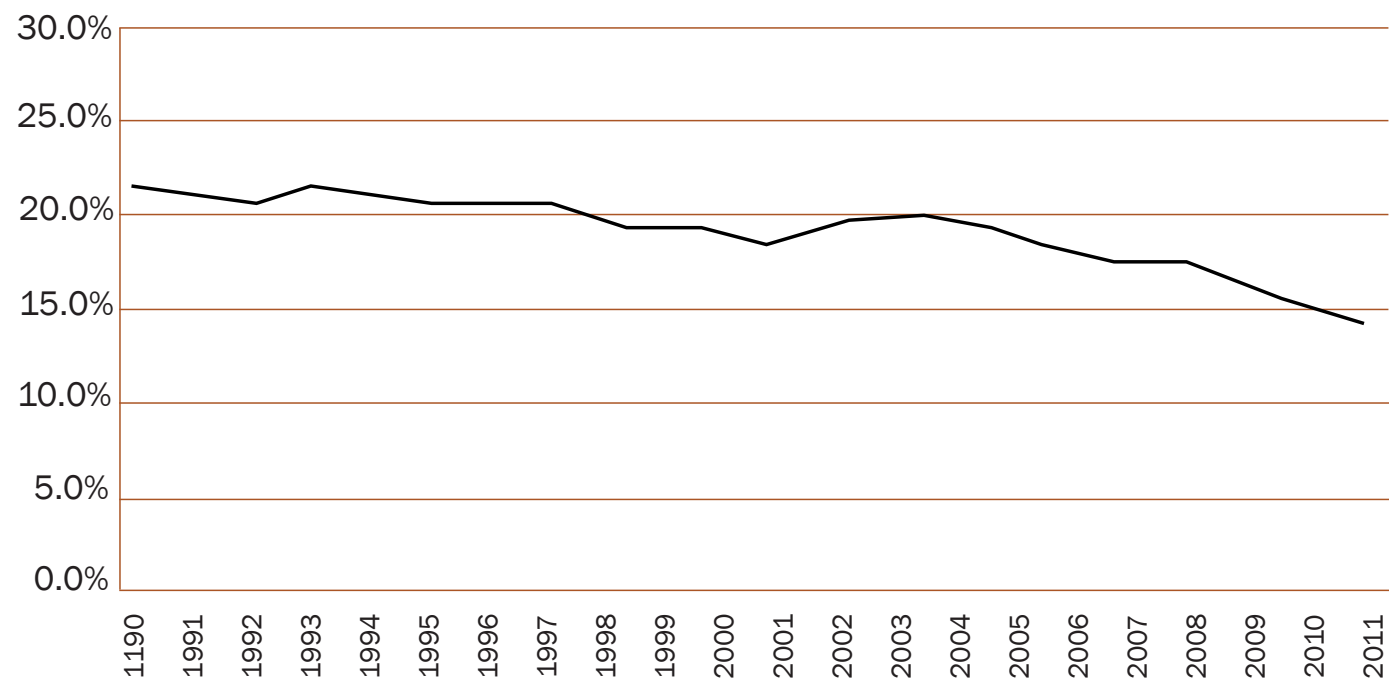




\section{Datos Generales de la Población Hondureña}

\begin{tabular}{lr}
\hline POBLACIÓN GENERAL & 8.041 .654 \\
\hline MUJERES & 4.094 .539 \\
\hline HOMBRES & 3.947 .115 \\
\hline DESEMPLEO & 133,737 \\
\hline SUBEMPLEADOS & $1,600,000$ \\
\hline COSTO CANASTA BÁSICA & $12,000.00$ \\
\hline SALARIO MINIMO ACTUAL & $5,500.00$ \\
\hline
\end{tabular}

** No cubren la canasta básica

Más de tres millones

DESEMPLEO

SUBEMPLEADOS

COSTO CANASTA BÁSICA

SALARIO MÍNIMO ACTUAL

NO CUBREN LA CANASTA BÁSICA

** Según proyecciones del INE el 2015 la población será de 9 Millones de habitantes
133,737

$1,600,000$

$12,000.00$

$5,500.00$ 


\section{Poblacion de 15 Años y mas, tasa de analfabetismo por año Total Nacional}

\begin{tabular}{lllll} 
& & \multicolumn{1}{c}{ TOTALES } & & \\
Año & Total & Alfabeta & Analfabeta & Tasa Analfabetismo \\
1990 & 2482,016 & 1852,226 & 629,790 & $25.4 \%$ \\
1991 & 2598,927 & 1994,888 & 604,039 & $20.8 \%$ \\
1992 & 2711,011 & 2147,943 & 563,068 & $21.4 \%$ \\
1993 & 2858,830 & 2246,935 & 611,895 & $20.2 \%$ \\
1995 & 3021,191 & 2411,982 & 609,209 & $20.2 \%$ \\
1996 & 3149,998 & 2508,888 & 641,110 & $20.4 \%$ \\
1997 & 3231,825 & 2573,409 & 658,416 & $20.4 \%$ \\
1998 & 3326,966 & 2690,936 & 636,030 & $19.1 \%$ \\
1999 & 3409,444 & 2753,020 & 656,424 & $19.3 \%$ \\
2001 & 3708,731 & 3015,423 & 690,308 & $18.7 \%$ \\
2002 & 3847,542 & 3079,664 & 767,879 & $20.0 \%$ \\
2003 & 3949,154 & 3168,209 & 780,945 & $19.8 \%$ \\
2004 & 4178,585 & 3406,913 & 771,456 & $18.5 \%$ \\
2005 & 4323,954 & 3557,179 & 766,775 & $17.5 \%$ \\
2006 & 4482,190 & 3698,856 & 783,335 & $17.5 \%$ \\
2007 & 4635,571 & 3822,272 & 813,298 & $17.5 \%$ \\
2008 & 4839,675 & 4036,914 & 802,761 & $16.6 \%$ \\
2009 & 5014,564 & 4230,981 & 783,583 & $15.6 \%$ \\
2010 & 5232,118 & 4406,681 & 825,436 & $15.8 \%$ \\
2011 & 54008,451 & 4603,852 & 804,600 & $14.9 \%$ \\
\hline & & & & \\
\hline
\end{tabular}

** Fuente Encuesta de Hogares de popósitos Multiples Mayo 1190-2011

\section{CAPITULO V}

\section{CONSECUENCIAS Y EFECTOS DEL ANALFABETISMO}

Las consecuencias del analfabetismo se reflejan en tres aspectos: social, político y económico. Es un problema social grave, desde luego que es factor de exclusión y marginación, cuyas raíces se hunden en las profundas desigualdades sociales, por lo cual este factor está estrechamente ligado a los otros dos aspectos. Por ello, es necesario analizar estas consecuencias a partir de una visión socio-política y socioeconómica. Pero, también, es un factor que conspira contra nuestras posibilidades de desarrollo y de fortalecimiento de nuestra capacidad competitiva que, por última instancia, depende del nivel educativo promedio de nuestra población. 
El analfabetismo trae consigo consecuencias de diferentes índoles. El desarrollo de Honduras sería más eficaz si en el país no existiera un alto porcentaje de individuos analfabetos, pues esto retrasa el desarrollo a nivel de la región, afectando también a nivel de todo el país. Desde el punto de vista socio-político, el analfabeto, no puede entender cuáles son sus derechos, debido a lo cual es marginado por la sociedad. Esto también, es motivo para aprovecharse de su ignorancia y explotarlo.

En lo que respecta al aspecto socio-económico, el conocimiento tiene un papel decisivo en los procesos productivos; entonces, si no existe una buena educación, se estaría impidiendo que los niños, jóvenes y adultos a nivel nacional puedan desarrollar sus capacidades intelectuales y humanas adecuadamente.

Nuestro país tiene la necesidad de una reforma en los tres aspectos desarrollados. El analfabetismo es, por tanto, un factor que conspira contra las posibilidades de desarrollo y de fortalecimiento de nuestras capacidades competitivas. Esto como consecuencias de una cuestión humanitaria y de justicia social que depende de la decisión política de los gobiernos. Por otro lado, se requiere una visión más realista la cual se ha contemplado en el plan de nación actual con visión de progreso y desarrollo hasta el año 2035, pues la alfabetización constituye también un factor determinante para lograr el crecimiento económico y la cohesión de las sociedades. Por ello, nuestro deber como hondureños es cambiar, para bien, cada factor que hace que el analfabetismo sea un problema en nuestra patria.

La relación analfabetismo - alfabetización podrá modificarse positivamente si existe la voluntad política de los gobiernos locales, municipales y estatales para intervenir y si se trabaja en sistema un conjunto de factores sociales, educativos y pedagógicos. La alfabetización es ante todo un fenómeno social-educativo y está vinculado estrechamente a otras esferas en el que el hombre se manifiesta de ahí que se requiera de un enfoque integrador para lograr resultados favorables.

\section{CONCLUSIONES}

- Llegamos a la conclusión de que el analfabetismo es un problema que afecta en mayor grado a la clase social más baja que no accede a una educación básica de nivel inicial. Las consecuencias de éste se agravan día a día y el Estado no toma suficientes medidas para combatirlo. En Honduras la zona urbana marginal es la más afectada después de la zona rural.

- En efecto, el problema del analfabetismo puede agravarse en lugar de mejorar, si al incrementarse la población en municipios, aldeas caseríos, no aumenta también, y 
de forma proporcional, el número de escuelas y maestros. Que eso es muy frecuente y bien marcado en la población.

- Si analizamos haciendo un enfoque a la problemática expuesta en la presente investigación, podemos considerar al analfabetismo como un fenómeno de carácter social directamente vinculado a la pobreza, que tiene sus fuentes en desigualdades sociales.

- Aun y con todos los retos y los compromisos emprendidos por el Estado, el Sector Educación y sector Empresarial a nivel nacional e internacional, la tendencia prevaleciente demuestra que el analfabetismo se reproduce como fenómeno social de forma creciente lo que hace necesario la búsqueda de políticas y nuevas estrategias no convencionales para su erradicación o disminución.

\section{RECOMENDACIONES}

- Debería de mejorarse el sistema educativo, y obligar a cada núcleo familiar a tener una educación primaria como base, multando a los irresponsables que permiten el abandono de sus hijos a las aulas de clases por cualquier situación.

- Tanto las instituciones públicas como privadas deberían de implementar como requisito primordial obligatorio para apartar el desempleo, haber cursado su primaria completa con ello disminuiremos esa alta tasa de $19.5 \%$ de hondureños que no saben leer ni escribir $(1,700,000)$ personas.

- Implementar equipos tecnológicos en el sistema educativo personalizado donde se cubra inmediatamente una investigación documental y de campo al número de personas por departamento.

- En el corto y mediano plazo, el país tiene que mantener políticas y programas emprendedores encaminados a lograr la universalización del ciclo de educación básica. Esto puede lograrse parcialmente mediante aumentos de eficiencia en la distribución de los recursos destinados a la educación, así como de inversiones deliberadas en programas dirigidos a poblaciones que tienden a permanecer por fuera del sistema, bien por su ubicación geográfica, por condiciones socioeconómicas y culturales, así como el desplazamiento forzado

- Alcanzar un mejor rendimiento y disminuir las tasas de ausentismo, deserción y repitencia. Este es un esfuerzo al que deben contribuir todos los actores de la sociedad. Es del conocimiento de todos el hecho de que a mayor cantidad de años 
de escolaridad (sin repitencia) mayores las probabilidades de que un país alcance mejores índices de desarrollo. El aumento de cobertura con calidad en la educación preescolar y la Educación General Básica (hasta el noveno año) debería ser una meta en la que se evidencia un esfuerzo intersectorial, esto es, Educación y Salud, con el apoyo de las diferentes secretarias vinculantes con educación.

- Tomar las investigaciones realizadas por las distintas fuentes y medios de difusión, y analizarlas exhaustivamente cada uno de ellas por los entendidos en materia de educación y una vez analizadas y seleccionadas convocar a los mismos para que puedan dar los aportes que de acuerdo al logro de resultados han obtenido en sus tareas investigativas y previa convocatoria integrar a cada investigador, como autores de las investigaciones seleccionadas, para que se inicie un programa de erradicación en contra del analfabetismo en nuestro país tomando en cuenta todos los factores que han sido fundamentales para el agravio del mismo, dejando de cometer los errores de siempre por estar copiando programas que en otros países han dado excelente resultado, pero se han enfocado en la problemática de su país, pues debe tomarse en cuenta que cada región tiene costumbres, tradiciones y forma de vida de acuerdo a las necesidades de la misma, por lo tanto no podemos copiar modelos que lo único que nos proporciona es un fracaso mayor a causa de la buena intención que tiene una minoría. Porque los políticos de turno (que solo abusan del poder que el pueblo les ha delegado para que les represente) creen que solo es de aprobar y obligar el cumplimiento de una norma sin medir las consecuencias.

\section{BIBLIOGRAFIA}

- Datos del programa para el desarrollo de las Naciones Unidas (PNUD) 2010 - Información del Centro de Documentación del Instituto de Investigación Nacional (INE). 2011

- Comisión Nacional para el Desarrollo de la Educación Alternativa no Formal( CO NEANFO)

- Articulo "El analfabetismo en Honduras" (Diario la Prensa hn. )

- Centro de documentaciòn estadístico de la Secretaria de Educación

- Encuesta Permanente de Hogares de Propósitos Múltiples

- Decreto \# 131 Diario Oficial la Gaceta.

- Constitución de la República 1982

- Diccionario Universal

www Honduras laboral.org. 